\title{
REVISTA
}

Revista Educación

ISSN: 0379-7082

ISSN: 2215-2644

revedu@gmail.com

Universidad de Costa Rica

Costa Rica

\section{La metacognición como factor de desarrollo de competencias en la educación peruana}

Moreno Muro, Juan Pablo; Arbulú Pérez Vargas, Carmen Graciela; Montenegro Camacho, Luis

La metacognición como factor de desarrollo de competencias en la educación peruana

Revista Educación, vol. 46, núm. 1, 2022

Universidad de Costa Rica, Costa Rica

Disponible en: https://www.redalyc.org/articulo.oa?id=44068165006

DOl: https://doi.org/10.15517/revedu.v46i1.43724

\section{(c) (1) $\Theta$}

Esta obra está bajo una Licencia Creative Commons Atribución-NoComercial-SinDerivar 3.0 Internacional. 


\section{La metacognición como factor de desarrollo de competencias en la educación}

peruana

Metacognition for Skills Development in Peruvian Education

Juan Pablo Moreno Muro

Universidad César Vallejo, Perú

DOI: https://doi.org/10.15517/revedu.v46i1.43724

mmurojp@ucvvirtual.edu.pe

Redalyc: https://www.redalyc.org/articulo.oa?

iD https://orcid.org/0000-0002-5236-7520

Carmen Graciela Arbulú Pérez Vargas

Universidad César Vallejo, Perú

carbulu@ucvvirtual.edu.pe

(iD https://orcid.org/0000-0002-8463-6553

Luis Montenegro Camacho

Universidad César Vallejo, Chiclayo, Perú

lmontenegroc01@gmail.com

iD https://orcid.org/0000-0002-8696-5203

Recepción: 20 Septiembre 2020

Aprobación: 11 Febrero 2021

\section{RESUMEN:}

El objetivo fue analizar las evidencias científicas sobre las características esenciales de la metacognición y sus ejes metodológicos como factor de desarrollo de competencias en la educación peruana. Se aplicaron métodos teóricos y se efectuó un análisis documental basado en la cartografía conceptual. Entre junio y agosto de 2020, se realizó la búsqueda, utilizando Google Scholar y Scopus, de publicaciones para el período comprendido entre 2000 y 2020, en español, inglés y portugués. Se incluyeron todos los estudios directamente relacionados con el problema y objetivo del estudio. En primer lugar, se analizaron veinticinco publicaciones científicas acerca de las características esenciales de la metacognición con base en el análisis del rol que corresponde al autoconocimiento en la configuración del proceso metacognitivo. Éste consiste en facilitar la caracterización de cada uno de los procesos que deben planificarse y regularse para mejorar continuamente la calidad de los aprendizajes. En segundo lugar, se profundizó la fundamentación sobre la importancia de la metacognición en el desarrollo de competencias. Se concluyó que es necesario integrar, en la definición de metacognición, la comprensión y dirección, por parte del alumnado; la dinámica holística entre motivación, voluntad, inteligencia, memoria, creatividad, modelación, desempeño, socialización y autovaloración cuando se resuelven problemas del contexto. Del mismo modo, se requiere profundizar en el análisis del autoconocimiento y su efecto en la calidad de los aprendizajes, por lo que se recomienda elaborar nuevas propuestas de modelos metodológicos basados en el enfoque socioformativo complejo.

Palabras clave: Desarrollo de competencias, Metacognición, Enfoque socioformativo, Gestión autónoma del aprendizaje, Autoconocimiento.

\section{Abstract:}

This study analyzes scientific evidence delineating essential features of metacognition and associated methodological considerations for skills development in Peruvian education. Various theoretical methodologies were used for document analysis based on a conceptual outline. A document search on was conducted on Google Scholar and Scopus from June through August, 2020 for articles published from 2000 to 2020, in Spanish, English and Portuguese which were directly related to the objective of the study. Afterwards, a total of twenty-five scientific publications on essential aspects of metacognition were examined according to the role of self-awareness in configuration of metacognitive processes, which consisted in describing of each process to be planned and regulated with the ultimate goal being to improve the quality of learning with emphasis on metacognition for skills development. According to the conclusion, for students to better understand and manage the definition of metacognition, it is necessary to include holistic dynamics such as motivation, will, intelligence, memory, creativity, modeling, performance, 
socialization and self-evaluation, when solving problems. There is also a need for greater in-depth analysis and its effect upon learning which includes, elaboration of new proposals for methodological models based on the complex socioformative approach. KEYwoRDs: Skills Development, Metacognition, Socio-formative Approach, Autonomous Learning Management, Selfknowledge.

\section{INTRODUCCIÓN}

En el siglo XXI, la dinámica del desarrollo humano ha configurado escenarios tan complejos como las dimensiones en las que la persona manifiesta su naturaleza. El esfuerzo por adecuar el entorno natural y los procesos sociales a sus necesidades e intereses ha implicado una sucesión de cambios que, en el proceso autopoiético de la sociedad, configura la necesidad de desarrollar la autodirección del aprendizaje.

En ese sentido, la mejora continua de la educación resulta impostergable si se pretende garantizar su calidad. En el presente caso, por la importancia de la metacognición en el proceso de aprendizaje, el objetivo del estudio es definir sus características esenciales y precisar sus ejes metodológicos para el desarrollo de competencias.

Para ello resulta pertinente tener un marco conceptual básico, a cuyo efecto, de acuerdo con Tobón (2015a), se entiende por competencia la actuación de la persona en un marco ecológico, esto según sus necesidades y motivaciones personales, problemas del contexto, y actitud proactiva frente a nuevos desafíos.

Con esa perspectiva se busca analizar las evidencias científicas sobre cuáles son las características esenciales de la metacognición y sus ejes metodológicos para el desarrollo de competencias.

Un antecedente importante a considerar, que justifica el estudio por la actualidad de la problemática, es el informe del Programa para la Evaluación Internacional de las y los Estudiantes (PISA, por sus siglas en Inglés) de 2018, que evidencia para Perú el reto de elevar el nivel de desarrollo de las competencias, porque la evaluación de lectura, ciencias y matemáticas se ubica por debajo del promedio de los países de la Organización para la Cooperación y Desarrollo Económico (OCDE) (Schleicher, 2019). Así lo ha constatado el mismo Ministerio de Educación (MINEDU) al aplicar su evaluación censal (ECE) en el mismo año.

Las causas pueden identificarse en varios de los componentes del proceso didáctico. Con la aplicación de un enfoque holístico de análisis, basta que uno de ellos fuera deficiente para que no se logren resultados satisfactorios. Sin embargo, el análisis del Diseño Curricular Nacional revela congruencia entre los principios, fines y metas de la educación básica, por lo que resulta lógico estudiar el proceso didáctico escolar en su conjunto como fuente de tales causas.

Por la observación sistemática del proceso, se detectan deficiencias significativas en la aplicación de estrategias de enseñanza-aprendizaje que enfaticen en el uso pertinente de la metacognición para definir metas de aprendizaje y la organización estratégica para lograr y garantizar la mejora continua del proceso de aprender; lo anterior constituye una calidad baja en el desempeño del estudiantado. Se evidencia, asimismo, un bajo nivel de sensibilidad en las actitudes y prácticas estudiantiles en cuanto a asuntos ambientales y reconocimiento de la diversidad y multiculturalidad; también, una deficiencia en el desarrollo del pensamiento crítico.

Al analizar a profundidad el rol de estos factores en el proceso de formación-aprendizaje, como lo denomina Motta (2002), se observa que su integración con factores externos configura una competencia esencial: la gestión autónoma del aprendizaje. En la distinción entre competencias específicas y transversales, resulta propio reconocer el carácter transversal de la metacognición por el rol fundamental en todo tipo de aprendizaje.

Se constata, también, la vigencia de enfoques y prácticas de la escuela tradicional, por el énfasis en la reproducción de contenidos. Se evalúan los resultados de la memorización de información y de hechos, y muy 
escasamente la solución de problemas del contexto. Las características descritas identifican a un profesorado concentrado en transmitir determinada información a receptores pasivos.

Sobresale, en el alumnado, la inadecuada motivación, factor trascendente en la gestión autónoma del aprendizaje, ya que una motivación eficaz activa e integra otros procesos cognitivos: voluntad, inteligencia, memoria, creatividad, modelación, socialización, desempeño, autovaloración.

Debe agregarse el uso, muchas veces inadecuado por parte del alumnado, de la tecnología y los distractores característicos de cada etapa de desarrollo, los cuales pueden ser obstáculos para la consecución de las metas estudiantiles.

En el marco de esta problemática se aprecia la debilidad en la aplicación de la metacognición. La población estudiantil prefiere instrucciones específicas para eludir las tareas propias de la investigación en la construcción del aprendizaje y no se percibe que utilicen - en ese proceso- estrategias que faciliten y consoliden la gestión autónoma del aprendizaje.

Sin embargo, se destaca la tendencia a organizar y desarrollar el proceso didáctico bajo el enfoque socioformativo. Se acepta la urgencia de desarrollar competencias genéricas como el trabajo colaborativo, aprender a aprender, capacidad de análisis-síntesis y las específicas de su formación; aun así, este interés aún dista mucho de logros satisfactorios.

Las competencias enumeradas se integran en una sola: gestión del aprendizaje de manera autónoma. Esta reflexión es necesaria porque permite comprender la integración de los cuatro saberes planteados por Delors (1999): conocer, hacer, ser, vivir en conjunto; es decir, aunque algunas personas se desarrollan predominantemente en determinada área curricular, requieren, sin embargo, la aplicación de lo que corresponde a otras; esto obliga un enfoque holístico complejo, si se pretende un estudio idóneo.

Debe resaltarse la cantidad y calidad de los procesos que debe conocer el alumnado para elaborar estrategias que le ayuden a dirigir eficazmente su interacción con los factores externos.

En la dimensión teórica, el estudio se fundamentó, básicamente, en el Enfoque Socioformativo Complejo (ESC) (Tobón, 2015a, Tobón et al., 2015), según el cual se requieren las condiciones esenciales para una formación idónea de las competencias, esto mediante la correspondencia entre la educación y la dinámica social del contexto en todas sus dimensiones: económica, política, histórica, etc. La tendencia a aplicar este enfoque crece al ritmo de las investigaciones y publicaciones relacionadas; sin embargo, resulta necesario profundizar en el análisis del autoconocimiento —dimensión esencial de la metacognición-y su efecto en la calidad de los aprendizajes, lo que justifica nuevas investigaciones que aporten modelos metodológicos innovadores con esa finalidad.

El estudio está alineado con la perspectiva de formación integral, que se destaca en el enfoque de la socioformación, definida como:

un marco de reflexión-acción educativo que pretende generar las condiciones pedagógicas esenciales para facilitar la formación de personas íntegras, integrales y competentes para afrontar los retos-problemas del desarrollo personal, la vida en sociedad, el equilibrio ecológico, la creación cultural-artística y la actuación profesional-empresarial, a partir de la articulación de la educación con los procesos sociales, comunitarios, económicos, políticos, religiosos, deportivos, ambientales y artísticos en los cuales viven las personas, implementando actividades formativas con sentido (Tobón et al., 2015, p. 43).

\section{Metodología}

El estudio fue cualitativo (Gómez et al., 2015), con perspectiva hermenéutica, con base en los siguientes métodos: histórico-lógico, inducción-deducción, abstracción-concreción, análisis-síntesis y sistémicoestructural. El propósito de la revisión bibliográfica, como lo plantean Guirao et al. (2008), fue identificar y analizar información sobre la situación actual del conocimiento científico de un tema específico, en este caso, la metacognición como factor de desarrollo de competencias, sobre la que se elaboró una síntesis sistemática (Reyes, 2020). 
Con base en la cartografía conceptual (Tobón, 2015b), se partió del análisis de las evidencias científicas más relevantes acerca de la definición de metacognición y su rol en el desarrollo de competencias. Los documentos se analizaron siguiendo una ruta epistemológica que va desde el concepto de personas autoras hasta su aplicación en casos prácticos.

Se efectuó la búsqueda de publicaciones - para la cual se utilizó Google Scholar y Scopuscomprendidas entre los años 2000 y 2020. La búsqueda se realizó entre junio y agosto de 2020. El principal criterio para la elección de los metabuscadores fue su reconocimiento internacional y la cantidad de resultados que ofrece cada uno. Se utilizaron las palabras clave: metacognición. desarrollo de competencias. Se obtuvo, en la búsqueda por metacognición y desarrollo de competencias, 38600 resultados, en Google Scholar; y en Scopus, 45 resultados para metacognicióny 92 para metacognición y desarrollo de competencias.

Como criterios de inclusión, se consideró la relación directa de la información identificada con el problema y objetivo del estudio, publicaciones en español, inglés y portugués entre los años 2000 y 2020 ; se excluyeron las que fueran muy específicas a un nivel educativo, área o disciplina, en atención al objetivo del artículo. Aplicados los criterios de exclusión, se seleccionaron solamente 25, considerados esenciales y suficientes para el objetivo del estudio. Se analizó con profundidad epistemológica y se sistematizó la información teórica sobre la metacognición para identificar y subsanar los vacíos semánticos, para lo que se adoptó una nueva postura teórica en el contexto de los enfoques y paradigmas vigentes.

Se asumieron cinco categorías de análisis para la realización del estudio. En cada caso se formuló una interrogante estructurada según las dimensiones consideradas para su análisis, como se indica a continuación.

1. Comprensión del término. ¿Cuál es la etimología del término metacognición y su definición actual?

2. Extensión del término. ¿A qué clase inmediatamente mayor pertenece la metacognición?

3. Caracterización. ¿Cuáles son los elementos esenciales que identifican a la metacognición y cuáles son sus características fundamentales?

4. Relación con otros procesos. ¿Qué aspectos esenciales vinculan a la metacognición con el desarrollo de competencias?

5. Metodología. ¿Cuáles son los criterios metodológicos básicos para el desarrollo y uso eficaz de la metacognición?

Cuatro fueron las fases del estudio. Primera, determinación de los criterios de búsqueda de fuentes. Esta se realizó principalmente en Scopus y Google Scholar. Se incluyeron todos los estudios directamente relacionados con el problema y objetivo del artículo. Las palabras clave fueron: desarrollo de competencias, metacognición, socioformación, gestión autónoma del aprendizaje y autoconocimiento. Se excluyeron los trabajos muy específicos a un nivel educativo, área o disciplina, en congruencia con el título y el objetivo del artículo, los publicados antes del 2000 o después del 2020 y los escritos en idioma distinto al español, inglés o portugués.

En la segunda fase se establecieron los criterios de selección de las fuentes. 1) relación de pertinencia con las palabras clave; 2) la proporción entre fuentes primarias y secundarias fue de dos a uno; 3) ambos tipos de fuentes debían cumplir los estándares internacionales de identificación; para artículos científicos, estar publicados en revistas indexadas; 4) en el caso de otros documentos, contener información precisa para su identificación (autoría, año, origen). Se puso énfasis en el análisis de la literatura más relevante para comprender cada eje del concepto.

La tercera fase fue la selección de documentos esenciales. Con la aplicación de los criterios establecidos, se seleccionaron veinticinco fuentes directamente relacionadas con el problema de investigación: ocho, específicamente sobre competencias; quince, con énfasis en el proceso metacognitivo o en el aprendizaje autorregulado; uno, sobre complejidad y educación; y uno, sobre pensamiento crítico. De ellos, ocho fueron 
libros, y diecisiete, artículos publicados en revistas científicas. Veinte en español, cuatro en inglés y una en portugués.

La cuarta fase, realización del análisis teórico, se cumplió en dos momentos: primero, el análisis teórico de cada uno de los cinco ejes clave asumidos; y segundo, el análisis de la relación de metacognición con el desarrollo de competencias. Ver Figura 1.

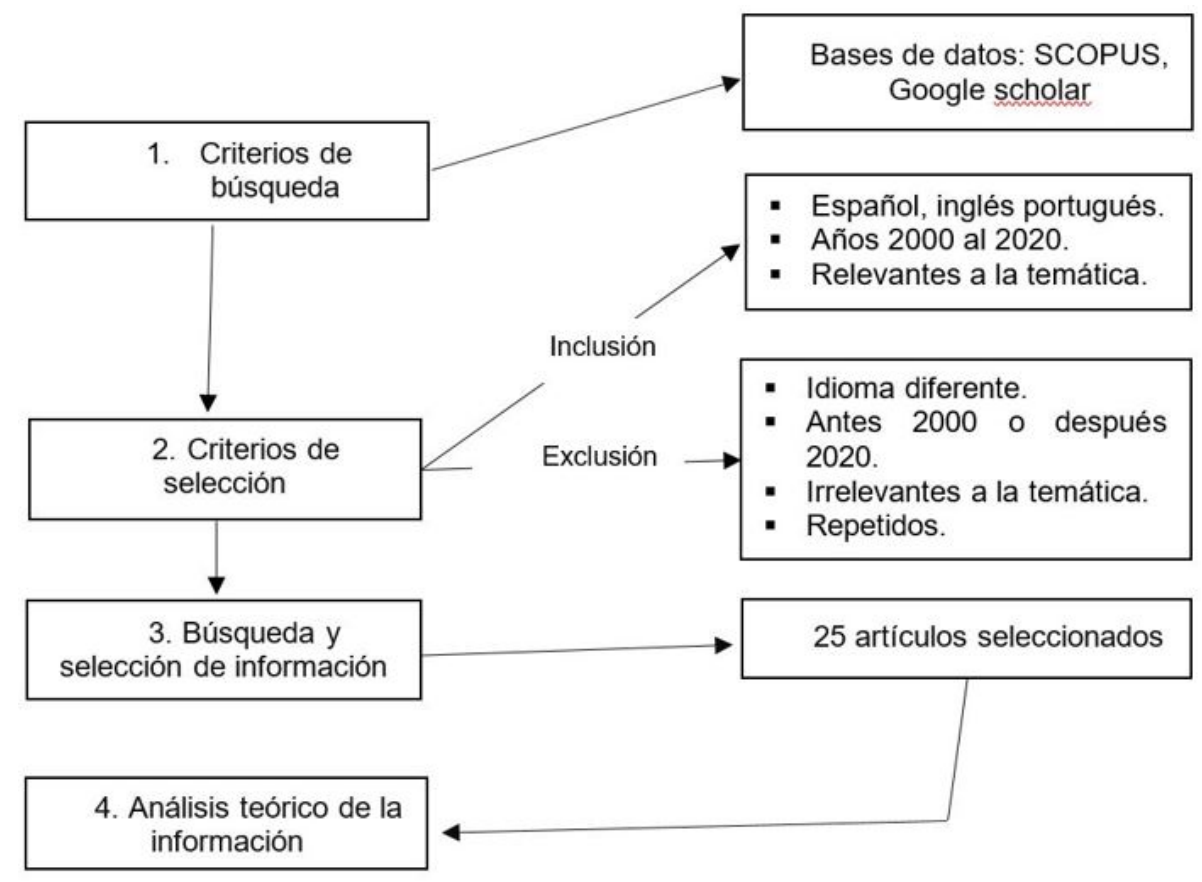

FIGURA 1.

Fases del estudio

Fuente: Elaboración propia

\section{Resultados}

Se presenta, a continuación, el análisis de los resultados de la revisión efectuada, los cuales atienden las cinco categorías establecidas en la metodología: comprensión del término, a partir de su etimología y definición actual; extensión del término, para delimitar la clase a la que pertenece; caracterización, a fin de precisar los elementos esenciales que identifican a la metacognición y sus características fundamentales; relación con otros procesos, para responder a la pregunta ¿Qué aspectos esenciales vinculan a la metacognición con otros procesos cognitivos?; y, finalmente, metodología, para determinar los criterios metodológicos básicos para el desarrollo y uso eficaz de la metacognición

\section{1. ¿Cómo se define la metacognición?}

El prefijo meta y el término cognición conforman el término metacognición. El primero tiene origen en el griego $\mu \varepsilon \tau \alpha$, que significa más allá (López, 1997). El segundo proviene del latín cognoscere, con el que se refiere a conocer; o de cognitı̌o (López, 1997), que se entiende como conocimiento.

Es abundante el número de investigaciones sobre temas relacionados con la metacognición: aprendizaje autodirigido (Shannon, 2008); enseñar para la autonomía (Monereo et al., 2001); metacognición y aprendizaje estratégico (Gonzáles, 2009); enseñanza de estrategias metacognitivas (Klimenko y Alvares, 2009); metacognición y autorregulación en entornos virtuales (Sierra, 2010); autorregulación del aprendizaje 
(Gaeta et al., 2012); uso de estrategias metacognitivas (Mato et al., 2017); aprendizaje autorregulado (Torrano et al., 2017); posible definición de metacognición para la enseñanza de las ciencias (Pérez y Gonzáles, 2020), etc. Como puede verse, la temática es objeto de estudio de muchas personas investigadoras, de manera que se mantiene vigente a través del tiempo.

En sus orígenes, las investigaciones sobre el tema en educación tuvieron como guía las propuestas de Flavell (1997) y Brown (1977), como se citó en Pérez y Gonzáles (2020), quienes superaron la postura de reducir el desarrollo de la metacognición a los mecanismos de la meta-memoria. Ambos consideran a la metacognición como el conocimiento de la cognición; es decir, de todos los procesos cognitivos, no solo de la memoria.

Para Flavell (1979, como se citó en Pérez y González, 2020), “el monitoreo de la cognición ocurre a partir de la acción e interacción entre 4 elementos: (1) El conocimiento metacognitivo, (2) Las experiencias metacognitivas, (3) Los objetivos de las tareas y (4) las acciones o estrategias utilizadas" (p. 386). El primer elemento incluye el componente netamente subjetivo, como insisten Botero et al. (2017), al reforzar la definición que se analiza como "la cognición sobre la cognición" (p.91), y es el centro de atención en el presente estudio, que, sin embargo, no alcanza a definir el detalle de este conocimiento.

Sobre la base de esta descripción, se ha planteado considerar dos dimensiones: el conocimiento metacognitivo y la regulación metacognitiva (Pérez y Gonzáles, 2020). En la definición de la primera, se precisa un poco más lo relativo a lo que el sujeto sabe —o cree saber- de los procesos cognitivos, sin llegar a definirlos o a detallar su dinámica, algo que, en el presente trabajo, se propone esencial para concretar con éxito la segunda dimensión, la regulación cognitiva.

Por su parte, Gonzáles (2009) afirma que

(la metacognición) alude al grado de conciencia y el conocimiento que las personas tienen sobre su propios procesos y eventos cognoscitivos; así como la habilidad para controlarlos, revisarlos y modificarlos en función de los resultados de la tarea intelectual que estén ejecutando (p. 133).

Se aprecia, en esta aseveración, mayor proximidad a la necesidad de conocimiento y control de los procesos cognitivos que interactúan en la metacognición, sin precisar, nuevamente, la dinámica de esa interacción.

En este mismo marco conceptual, en el análisis del aprendizaje, Pozo (2006, como se citó en Klimenko y Alvares, 2009) reconoce que implica la autorregulación como un proceso propio de la actividad de aprendizaje.

En complemento a lo planteado, para Klimenko y Alvares (2009), este tipo de aprendizaje requiere el desarrollo de la capacidad de aprender mediante la toma de conciencia y la adquisición del conocimiento sobre las propias capacidades, habilidades y características particulares, las cuales facilitan o dificultan el aprendizaje de determinadas tareas sobre los diferentes tipos de información y sus características. Asimismo, requiere de estrategias cognitivas que puedan emplearse con cada tipo de información y "aprender a regular su propio proceso de aprendizaje, supervisándolo y orientándolo de una manera independiente y autónoma" (p.17). Se observa coincidencia en la atención a las dos dimensiones propuestas por Pérez y Gonzáles (2020).

Siguiendo el análisis de los elementos que definen la metacognición, conviene tener en cuenta estudios sobre la autorregulación del aprendizaje, como el de Gaeta et al. (2012), quienes incluyen en su análisis el uso de estrategias de control -motivacional y emocional-, y encuentran que, cuando esto sucede, es mayor el compromiso del estudiantado hacia el aprendizaje y al empleo de estrategias de control cognitivo.

En la misma dirección, Torrano et al. (2017), por su parte, encontraron, entre los rasgos que caracterizan al alumnado que autorregula su aprendizaje, la actuación sobre, al menos, tres de los procesos: voluntad, atención y motivación. Descubrieron que este tipo de estudiantes aplica estrategias volitivas para evitar los distractores internos y externos, a fin de mantener su concentración, esfuerzo y motivación durante el proceso de aprendizaje; es decir, de desarrollo de competencias. 
La esencial importancia de estos procesos radica en su propia definición; la motivación, porque desencadena, impulsa y sostiene la dinámica generada por su interrelación; la voluntad, porque asegura la dinámica; y la atención, porque centra el foco de la conciencia en el objeto de aprendizaje. En el complejo proceso metacognitivo, puede entenderse que los tres factores señalados se integran, como complementan Gaeta et al. (2012). La Figura 2 ilustra lo analizado.

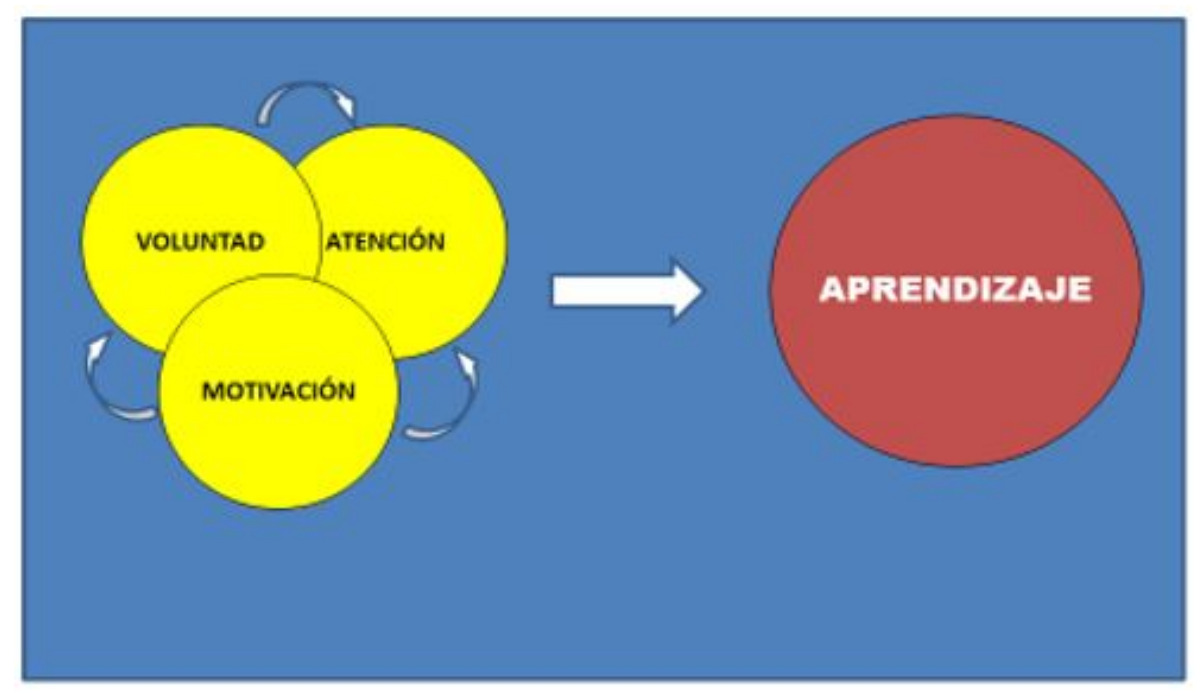

FIGURA 2.

Relación motivación, voluntad, atención y aprendizaje.

Fuente: Elaboración propia.

Con la misma lógica, y a partir del análisis de Gonzáles (2009), en interpretación de las personas autoras del presente artículo, la metacognición es el factor nuclear del aprendizaje, que se va definiendo a partir de la integración holística de los mencionados con los tres procesos siguientes: memoria — con sus funciones de fijación y evocación-, imaginación e inteligencia-ambas fundamentales en la modelación-, como se ilustra en la Figura 3. Al tratarse de una dinámica holística, la integración es incesante. 


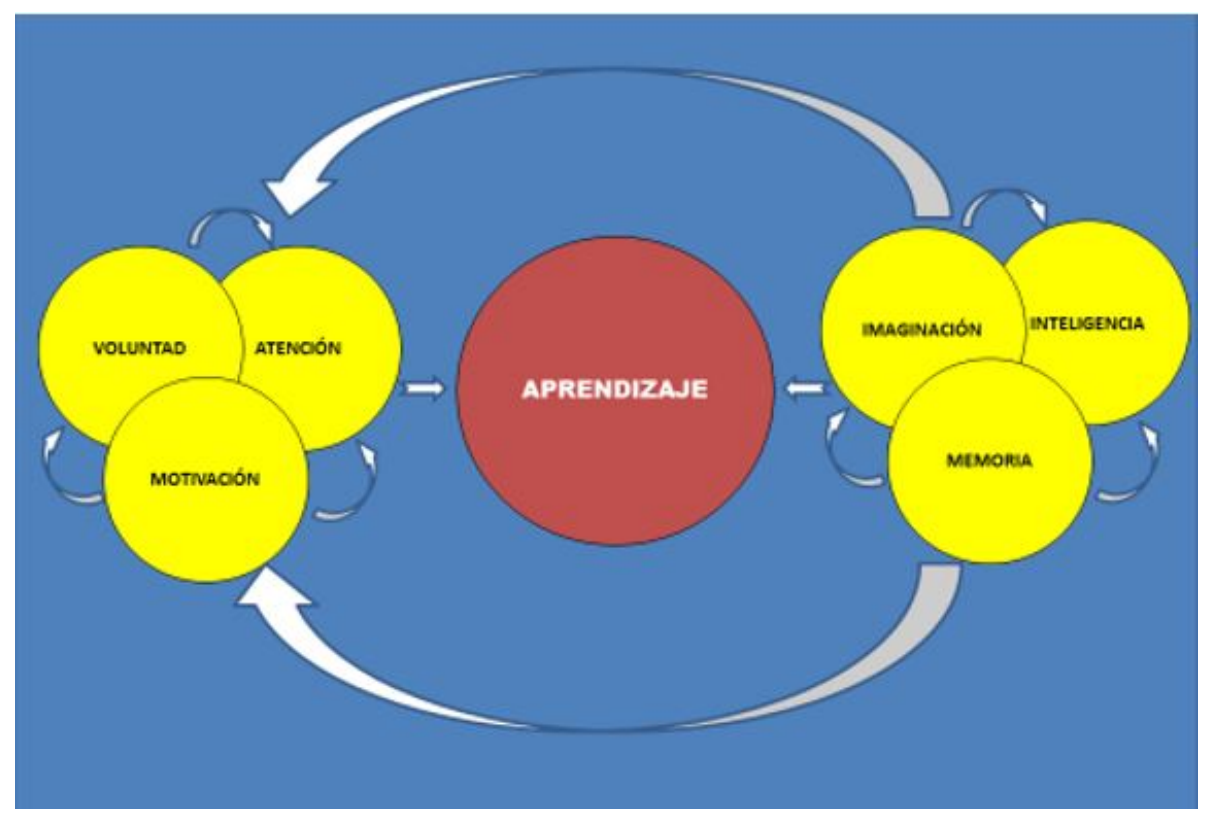

FIGURA 3.

Integración de motivación, voluntad y atención con memoria, imaginación e inteligencia en el proceso de aprendizaje.

Fuente: Elaboración propia

Finalmente, se produce la actuación en el contexto. Para ello, en interpretación de las personas autoras, a los procesos ya integrados se adhieren tres más: el desempeño, la socialización y la autovaloración, como puentes entre lo subjetivo y el contexto en el que se aplicará lo aprendido. Así analizados, se conciben como esenciales en la configuración de la metacognición. Figura 4. 


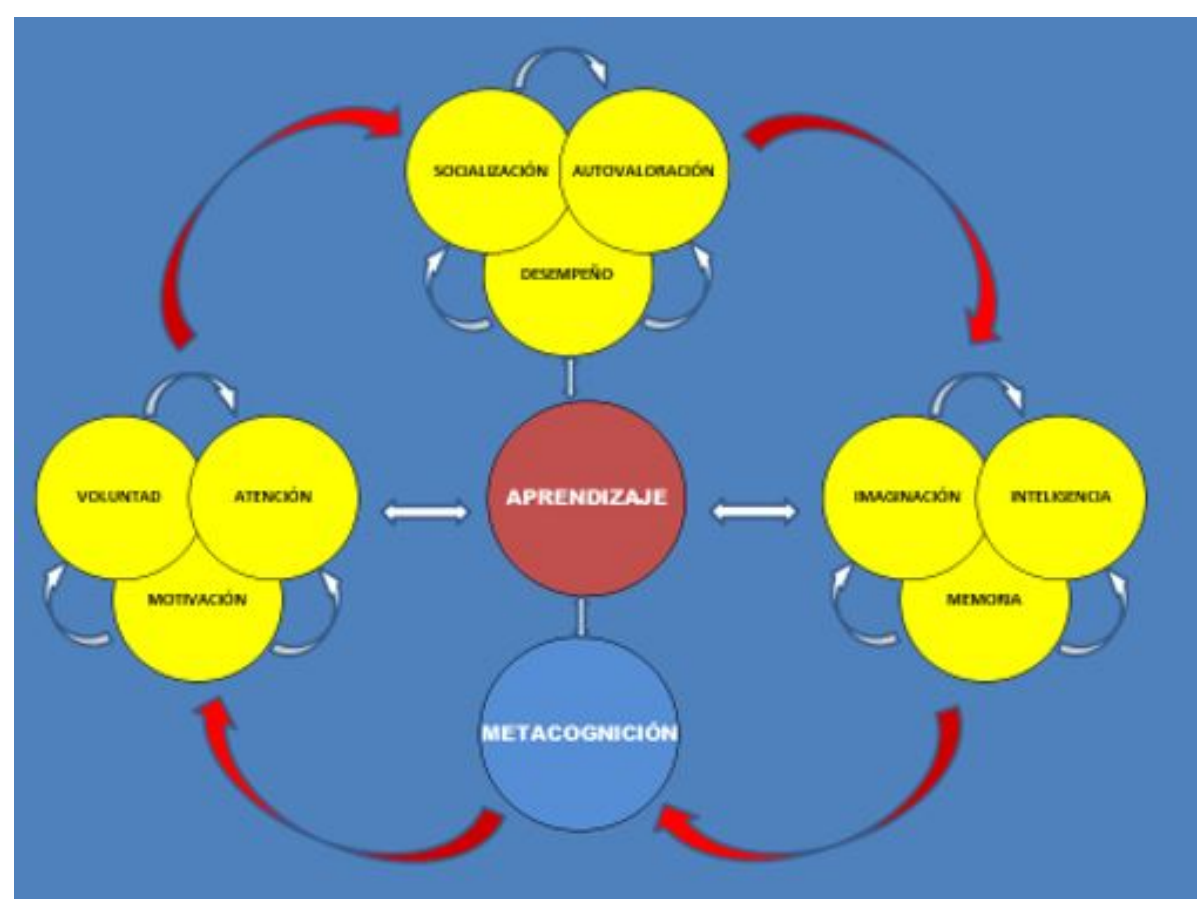

FIGURA 4.

Integración de varios procesos en la metacognición para definir el aprendizaje (desarrollo de competencias).

Fuente: Elaboración propia

En esta línea de análisis debe agregarse, además, que la modelación puede partir de elementos captados por los sentidos o de contenidos fijados y evocados por la capacidad mnémica para construir los conceptos, juicios y raciocinios, así como su relación entre sí como fundamentos de respuestas a la problemática del sujeto.

Asimismo, en la definición de metacognición se debe tener en cuenta, por su vigencia, planteamientos como los de Bolhuis (1996) y Garrison (1997, como se citó en Shannon, 2008), para quienes la autodirección integra el "manejo del contexto (que incluye el entorno social, los recursos y las acciones) y la automonitoreo (monitoreo, evaluación y regulación de sus estrategias cognitivas)” (p. 15).

Teniendo en cuenta estos dos componentes de la autodirección, puede colegirse que el desempeño y la socialización resultan objeto de autoconocimiento y autorregulación; es decir de la metacognición que, finalmente, se reinicia fortalecida a partir de la autovaloración.

En la definición asumida en el enfoque socioformativo (Tobón, 2013), se enfatiza un elemento adicional a los propuestos por investigadores e investigadoras precedentes: la mejora continua del desempeño, la que permite prevenir, reconocer y corregir los errores a tiempo. Así, se plantea y se supera el concepto tradicional enfocado en la toma de conciencia y la autorregulación.

En síntesis, con respecto a la primera categoría del análisis y por la coincidencia de elementos identificados en las definiciones revisadas, la metacognición puede definirse alrededor de la propuesta de Pérez y Gonzáles (2020), es decir, como el proceso complejo de conocer los procesos cognitivos — propios o de las demás personas - que comprende dos dimensiones: el conocimiento metacognitivo y la regulación metacognitiva. Es necesario destacar que en ambas se produce una dinámica de interacción entre factores internos (los procesos cognitivos) y externos (el contexto).

Finalmente, de lo analizado se deduce la necesidad de precisar, aún más, cómo puede promoverse el conocimiento y regulación de los factores internos para mejorar la calidad del aprendizaje. 


\section{Extensión del término}

Respecto a esta categoría del estudio, y con base en la información revisada en el apartado anterior, la metacognición es un componente esencial del aprendizaje, como lo sostiene Pozo (2006, como se citó en Klimenko y Alvares, 2009). En efecto, la complejidad del proceso de aprender requiere una especial arquitectura, cuya base está en el autoconocimiento de los diferentes procesos que intervienen, como la motivación y la voluntad, identificados por Torrano et al. (2017), sin que puedan excluirse varios más, como los descritos en el análisis de la definición que se ha efectuado.

En esta lógica de análisis, metacognición se ubica en la clase del término aprendizaje, tipificado ya como el proceso de desarrollo de competencias.

\section{Caracterización}

Nelson y Conner (2008, como se citó en Shannon, 2008) facilitan el análisis de esta categoría, al proponer como características de la metacognición: motivación, orientación a metas, autoeficacia, control y autorregulación.

$\mathrm{Al}$ analizar lo expuesto con atención, se observa coincidencia con la posición que se ha asumido respecto a la autorregulación y los procesos metacognitivos, en donde la motivación es un factor muy importante. El presente estudio busca analizar las bases para que el estudiantado autorregule con éxito su aprendizaje, como plantean Rovers et al. (2018), a partir de la autodirección de los procesos subjetivos del aprendizaje revisados — complementados por las personas autoras del presente artículo-; precisamente se inicia con la motivación como detonante, a la que se integran varios otros de manera sinérgica, dinámica e incesante.

Complementariamente, una tendencia que debe destacarse, por su efecto en los procesos metacognitivos, es la atención a la aplicación del pensamiento crítico, ya que involucra habilidades que consisten en analizar y evaluar argumentos. En este caso, para que el alumnado pueda construir el conocimiento, lo que debe entenderse, plantea Stenberg (como se citó en Wisdom y Leavitt, 2015), como los procesos y estrategias usadas para la solución de problemas y aprender nuevos conceptos.

Esta perspectiva es de particular importancia por su aplicabilidad en el proceso de gestión del aprendizaje -o ejercicio de la metacognición - por el propio alumnado, en la medida que le facilitará la toma de decisiones respecto a la multiplicidad de problemas que debe afrontar y resolver en su trayecto al logro de sus metas.

El análisis efectuado sobre la necesidad de que el estudiantado logre el uso del pensamiento crítico permite confirmar su importancia en la configuración, gestión y continua mejora de su aprendizaje de manera autónoma. Como destacan Palacios et al. (2017), se requiere diseñar y aplicar metodologías para lograr un razonamiento crítico en el alumnado, esto como base estructural del modelo de autogestión que elabore y aplique.

Debe subrayarse, sin embargo, la importancia del factor responsabilidad en la gestión autónoma del aprendizaje y que la asunción de responsabilidad - sobre un proceso tan complejo- solo puede concretarse con base en el pleno conocimiento de este; es decir, una total claridad de conciencia sobre sus motivaciones, dinámica, e impacto en la totalidad del ser, expresado en el desempeño y en la solución de los conflictos cognitivos, afectivos y volitivos que desencadena la dinámica de la vida anímica y del comportamiento humano.

En el presente estudio se asume el enfoque socioformativo complejo, en cuanto se orienta al desarrollo integral de la persona discente vía solución de problemas concretos de la sociedad, sustentados en sus propias vivencias (Tobón, 2015a). 
Con relación al proceso de metacognición, sin desconocer la importancia de lo planteado en el mismo enfoque, debe concentrarse la atención en la necesidad de que el estudiantado logre conciencia no solo de su actuación, sino también de los procesos subyacentes a tal actuación; es decir, se plantea que la autodirección de su actuación será auténtica en la medida que puedan comprender y gestionar los subprocesos cognitivos que configuran el proceso de aprendizaje.

En síntesis, el proceso metacognitivo ha de incluir, por un lado, la reflexión o toma de conciencia sobre los procesos internos y su dinámica; y, por el otro, la autorregulación para garantizar una mejora continua del aprendizaje, expresado en el desempeño del alumnado en un contexto determinado. Se busca considerar, como lo recomienda Morin (1999), la perspectiva multidimensional y compleja; el contexto y lo global.

\section{Relación con otros procesos.}

Con la finalidad de delimitar adecuadamente la comprensión y extensión del término metacognición y del concepto que expresa, conviene establecer su relación con algunos de los procesos esencialmente asociados a ella, ya formulados anteriormente y que, en conjunto, configuran el proceso de aprendizaje: en primer lugar, por su frecuente identificación, se analiza la relación con la motivación, reconocida ya como factor esencial en el proceso; y la memoria, como elemento clave en el proceso de pensar, para luego precisar su vinculación con la creatividad, la modelación, y la autoevaluación.

Cook y Artino (2016, como se citó en Rossi et al., 2020) entienden la motivación como "un proceso cuyo énfasis está en el logro de uno o más objetivos, y en el que importa tanto el inicio como la continuidad de la actividad necesaria para que se puedan alcanzar las metas que se hayan establecido" (p.257). Así entendida, la calidad de la motivación permite predecir el rendimiento, como se ha comprobado en estudiantes de educación primaria (Valle et al. 2015) y de primer nivel universitario (Thornberry, 2008). Sin embargo, estas constataciones no pueden considerarse una premisa de la que pueda deducirse que metacognición y motivación sean el mismo proceso, sino que, más bien, tributan en favor de su diferenciación.

Así entendida, la motivación, sin duda, cumple la función de activar la dinámica cognitiva del estudiantado en su proceso de aprender; por tanto, su efecto es previo, concurrente y posterior a este, como lo es el de la metacognición, por lo que podrían confundirse. Sin embargo, debe precisarse que la motivación no implica el autoconocimiento ni el monitoreo ni el control o evaluación de los productos del aprendizaje, componentes esenciales de la metacognición.

En relación con la memoria, esta sirve a la metacognición, con sus mecanismos de fijación, asociación y evocación de contenidos, tanto en la fase de reflexión, como en la de autorregulación, con fines de mejora continua de la calidad de los aprendizajes. Debe subrayarse, sin embargo, lo precisado por Pérez y Gonzáles (2020) en cuanto que la metacognición trasciende al concepto de meta-memoria.

Si bien su rol alcanza un espectro temporal del que trasciende el aprendizaje mismo, es evidente que la función mnémica es esencial en el proceso de metacognición y, por ende, del aprendizaje, pero no constituye la metacognición misma.

En este punto, conviene enfatizar la posición de Brown (como se citó en Pérez y Gonzáles, 2020), que considera a la metacognición como el conocimiento de la cognición y reconoce la participación de otros procesos cognitivos en ella, además de la meta-memoria, meta-atención y meta-aprendizaje. Por el análisis efectuado, se plantea que corresponden a los seis procesos complementarios precisados en el apartado 1 de este trabajo, referido a la definición de metacognición.

Por otro lado, a partir de la definición de los términos, la creatividad y la modelación, al igual que los ya analizados, constituyen factores esenciales en el conocimiento del proceso de aprendizaje; tanto más porque la creatividad - expresión de la imaginación- permite la modelación del objeto de conocimiento o aprendizaje a partir de la abstracción de sus características fundamentales y correspondiente creación de 
conceptos, juicios, argumentación y evaluación, que le permiten tomar posición respecto a su comprensión y definición.

$\mathrm{Al}$ respecto, es pertinente considerar la afirmación de Caeiro-Rodríguez (2018): "la Creación supone una vivencia en la cual el discente se proyecta en la acción creadora” (p. 163), sin perder de vista, por ello, que, en esencia, el aprendizaje constituye parte de una vivencia integral, en la que se funden, holísticamente, procesos tan complejos como el pensamien to y la comunicación, que contribuyen a la configuración de la personalidad de cada sujeto.

La modelación constituye la parte central del aprendizaje, en la que la percepción de nueva información y nuevas experiencias concretas se asocian con los contenidos previos conservados en la memoria. Surge, así, el concepto de integración de muchos procesos que, sinérgicamente, configuran la metacognición, y de cuya calidad dependerá, en gran medida, el aprendizaje.

A los procesos mencionados, sin ningún criterio de exclusión, debe agregarse la autovaloración, que, por este mismo concepto de sinergia, es inherente a todo el complejo proceso de metacognición y su resultado: el aprendizaje.

En la ruta metodológica asumida, en congruencia con el objetivo planteado, corresponde analizar, a continuación, la relación de la metacognición con el desarrollo de competencias.

Se han realizado numerosas investigaciones alrededor del concepto de competencias como se usa en el contexto educativo; y planteado muchas propuestas de modelos, estrategias y metodologías didácticas para su desarrollo en los diferentes niveles de los sistemas educativos del mundo. En este sentido, Díaz-Barriga (2006) articula las competencias al quehacer educativo y ubica su aplicación en el campo curricular.

Tobón (2015a) postula el enfoque socioformativo complejo (ESC), que incluye el pensamiento complejo. Enfatiza el rol social de la educación, porque se trata de preparar a la persona para su inserción social proactiva y eficaz, con base en su proyecto de vida. Este planteamiento complementa la perspectiva de la complejidad respecto a la sucesión de transiciones hacia la realización humana en el tejido de las relaciones entre el individuo y la sociedad (Tobón et al., 2010). Precisamente, en esta línea de análisis se asume tal enfoque, el cual "promueve abordar la formación humana integral como un sistema, y en esa medida busca identificar los ejes esenciales o nodos de la formación, los cuales orientan su estructuración y dinámica” (Tobón, 2015a, p. 25).

En el contexto de las competencias, con carácter referencial, merece mencionarse que, en el nivel superior, en complemento a la definición de competencias genéricas asumidas por el Ministerio de Educación de Colombia, Sepúlveda (2017) ha aportado propuestas para una mejor definición y desarrollo de las competencias genéricas, en las que resalta que las competencias transversales, al no ser específicas de un área, manifiestan valores útiles para diversas tareas y situaciones, no solo a nivel técnico, sino también a nivel práctico, lo que asegura la eficacia en el desarrollo de las actividades.

Según este concepto, las competencias genéricas son base de todo aprendizaje, pues incluyen capacidades cognoscitivas, críticas y de autocrítica; metodológicas, de sensibilidad; y destrezas tecnológicas, lingüísticas y sociales, fundamentales para el aprendizaje permanente. El análisis profundo de su aplicación práctica aproxima nuevamente al concepto de una sinergia entre todas las habilidades, destrezas y conocimientos involucrados.

Estos hallazgos, al relacionarlos con los procesos en otros niveles educativos, resultan interesantes y factibles de considerar válidos. Por ello, conviene destacarlos con relación a la metacognición desde la perspectiva de gestión del aprendizaje de manera autónoma — como se denomina en Perú oficialmente-, entendido como una competencia transversal y que sugiere la necesidad de que el estudiantado conozca y aplique tal metodología como parte de sus recursos de autodirección del aprendizaje.

Esta perspectiva resulta útil de aplicar en el proceso de gestión del aprendizaje por el propio estudiantado, en la medida que le facilitará la toma de decisiones respecto a la multiplicidad de problemas que debe afrontar y resolver en el trayecto hacia el logro de sus metas. 
Complementariamente, es ilustrativo el énfasis respecto a que el concepto de competencia — tal como lo concluye De Zubiría (2013) en su análisis de las posiciones de Chomsky, Piaget, Levi-Strauss y Al thusserexpresa las estructuras profundas en el lenguaje, si se trata de la competencia lingüística; en la inteligencia, si se trata de la competencia cognitiva; en las culturas, la economía y la sociedad, si se trata de competencias culturales, económicas, o sociales, respectivamente.

Si se asume este enfoque para el análisis del proceso formativo, en donde el estudiantado es el actor principal, debe aceptarse que, si se intenta garantizar la calidad del producto, es necesario promover en él la comprensión y gestión de los factores subjetivos que lo determinan, en interacción con el entorno específico.

Esta precisión es muy significativa en el presente estudio, cuyo objeto trasciende los aspectos factoperceptibles comprendidos en los desempeños aludidos - expresa o tácitamente- en muchas definiciones de competencias y se orienta, directamente, al análisis esencial de las estructuras y procesos subjetivos que configuran los saberes, capacidades, habilidades, destrezas y actitudes que se integran cuando los seres humanos resuelven sus actuaciones.

De acuerdo con Tobón (2008), metacognición y ética son dos de las seis dimensiones esenciales comprendidas en el enfoque socioformativo que inspira el presente estudio, en particular porque se refieren específicamente a los vacíos encontrados por las personas investigadoras, como la falta de énfasis en el autoconocimiento de los procesos subjetivos que configuran la metacognición, lo que plantea la necesidad de profundizar el estudio de la forma cómo se alcanza ese nivel de autoconciencia.

$\mathrm{Al}$ aplicar la perspectiva de la formación de personas integrales asumida en el enfoque socioformativo, los dos conceptos referidos - metacognición y ética- conforman un binomio que expresa el nivel de autoconciencia y dominio de los procesos que configuran la competencia y cuya dinámica determina su desarrollo. En efecto, Tobón et al. (2015), aluden a la socioformación como el enfoque orientado a facilitar la "formación de personas íntegras, integrales y competentes" (p.43), concepto precisado en la introducción del presente artículo, enfatizado por Tobón (2015a) respecto al enfoque: "el mismo que promueve abordar la formación humana integral como un sistema, y en esa medida busca identificar los ejes esenciales o nodos de la formación, los cuales orientan su estructuración y dinámica” (p.25).

Si bien la vida psíquica constituye una unidad que no puede dividirse, en el intento metodológico de identificar la arquitectura interna de tales procesos, el análisis de este artículo se limitó a comprender cómo el estudiantado, en búsqueda de la realización personal, vive, comprende y gestiona la motivación, voluntad, atención, inteligencia, memoria, imaginación, modelación, socialización, desempeño, autovaloración y los componentes de la metacognición, con una perspectiva holística que reconoce la integración dinámica entre ellos.

En búsqueda de las características esenciales de la metacognición y del proceso de aprendizaje, se ubica la motivación como punto de partida, como anotan Botero et al. (2017): "En este proceso, los estudiantes tienen la labor de hacer dinámica la relación entre los recursos cognitivos y motivacionales, para considerar las acciones que los coloque en el plano de lograr las metas indicadas" (p.92).

$\mathrm{El}$ análisis profundo del comportamiento humano permite hipotetizar una explicación a partir de los motivos que activan la voluntad para iniciar la dinámica subjetiva de todo proceso de aprendizaje -y de toda acción-. Sin embargo, muchos de los actos humanos se realizan de manera subconsciente; es decir, sin una clara conciencia de los motivos que los desencadenan ni de las capacidades que interactúan, lo cual puede resultar limitante para su autogestión; de ahí que motivación y voluntad deban entenderse en su innegable relación dialéctica como los factores iniciales del proceso de construcción de aprendizajes.

A los procesos anteriores - motivación y voluntad - se integra la inteligencia, para ordenar, dirigir y evaluar la actuación de la memoria y la creatividad en el proceso de socialización en que se concreta el aprendizaje propiamente dicho. 
Los desempeños basados en esos aprendizajes significativos, por la mediación de la autovaloración, realimentan y gratifican al ser, y establecen las bases de la realización personal, entendida como un estado de bienestar y satisfacción.

Consecuentemente, con base en los análisis efectuados en la categoría definición, debidamente ilustrados, se propone integrar en la definición de metacognición a la comprensión y dirección por parte del estudiantado, y la dinámica holística entre la motivación, voluntad, inteligencia, memoria, creatividad, modelación, desempeño, socialización y autovaloración cuando se resuelven problemas del contexto, para así generar la base de la autorrealización.

Se reconoce como esencial la dimensión metacognitiva en la construcción y desarrollo de las competencias o aprendizajes, lo que se logrará con éxito en tanto el alumnado comprenda y dirija, con una visión holística, los nueve procesos básicos descritos, mientras distingue cada uno de ellos en su relación con los demás y su interrelación, que conduce a la configuración de la competencia.

Lo expresado implica, a la vez, la necesidad de replantear la gestión del currículo desde la dimensión teleológica en la que se concibe la educación y que se pretende concretar a través de este, pero no solo para la educación básica, sino, también, en el diseño de la formación docente inicial en las facultades de educación (Sierra y Carrascal, 2005).

El marco teórico analizado refuerza la perspectiva teleológica de la educación formal; es decir, la formación integral de la persona como su finalidad (Tobón, 2008, Tobón et al., 2015). Las decisiones curriculares y didácticas deben basarse, por tanto, en esta perspectiva.

En este contexto, es pertinente la recomendación de De Zubiría (2013) respecto a que la asunción de un nuevo paradigma educativo, a partir del concepto de competencia, no puede concretarse si se mantienen "los mismos currículos, los mismos fines, contenidos, secuencias y textos, y guiadas por los mismos maestros de antes" (p. 20); es decir, el punto de partida para una revolución educativa es el cambio curricular.

El análisis efectuado compensa la insuficiente incidencia en el estudio teórico de la dinámica de los procesos subjetivos que configuran el aprendizaje, lo que ha motivado el análisis epistemológico de los conceptos de competencia y de metacognición, en busca de una comprensión más cabal de estos, y que sirva de base, a la vez, para una definición más precisa del proceso de gestión autónoma del aprendizaje.

\section{Metodología}

Como base para el análisis de este eje, conviene tener en cuenta las dimensiones que destacan en la definición de metacognición propuesta por Gonzáles (2009),

la primera dimensión alude al grado de conciencia y el conocimiento que las personas tienen sobre su propios procesos y eventos cognoscitivos; y la segunda, a la habilidad para controlarlos, revisarlos y modificarlos en función de los resultados de la tarea intelectual que estén ejecutando (p. 133).

En el presente trabajo puede constatarse que no se han identificado planteamientos sobre metodologías específicas que integren algunas estrategias, mucho menos para estudiantes peruanos. La búsqueda de una respuesta condujo a considerar la relación de interdependencia que se percibe en ambas dimensiones, lo que implica la necesidad de respetar su unidad para el planteamiento de una metodología; unidad que, como corresponde a todo proceso psicológico, es esencialmente original y resulta de la integración de todos los factores internos analizados, que configuran los estilos de aprendizaje: factores externos aportados tanto por la educación formal como por la espontánea.

Con esta visión de la complejidad de la metacognición, cuya génesis conduce hasta la misma personalidad, se ordena y manifiesta la vida anímica de cada sujeto. Se entiende que cada estudiante define sus propias estrategias, particularmente de acuerdo con su estilo de aprendizaje, sin eludir la responsabilidad del profesorado de sugerir una metodología general que pueda servirle de base. 
Por ello, en consideración de las definiciones y los análisis de las características propuestas que han sido objeto de la revisión, se proponen los siguientes procedimientos:

a. Reflexión inicial sobre los factores cognitivos y externos del proceso; b. planificación: fijación de metas, determinación de recursos, formulación de procedimientos, elaboración de cronograma; c. autodirección: formulación de compromiso, automonitoreo, autoevaluación del proceso, realimentación del proceso; d. socialización y. autoevaluación: valoración de resultados, realimentación; y f. reflexión final.

Así, se recomienda partir de una reflexión orientada por preguntas sugeridas al estudiantado, entre las que pueden incluirse: ¿cómo se produce mi aprendizaje?, ¿qué procesos intervienen en él?, ¿en qué consiste cada uno?, ¿qué relaciones puedo establecer entre ellos?, ¿puedo manejarlos a mi voluntad?, etc. Con esta base se puede pasar a la siguiente fase, la planificación de tareas y determinación de recursos necesarios para cumplirlas; y así sucesivamente a las siguientes, hasta finalizar con la reflexión final que, a su vez, significa el inicio de un nuevo ciclo.

Los cinco pasos constituyen una ruta que adquirirá las peculiaridades que identifican a cada estudiante; le permitirá definir y fortalecer su arquitectura psicosocial para lograr un aprendizaje significativo; en otras palabras, para desarrollar exitosamente las competencias previstas por la escuela o por el mismo estudiantado.

\section{Conclusiones}

En congruencia con el objetivo del artículo, la revisión de los artículos científicos seleccionados permitió identificar las siguientes características, que conducen a reconocer a la metacognición como factor de desarrollo de las competencias en cualquier proceso educativo formal, por tanto, de validez para la educación peruana:

1. La metacognición es un proceso multidimensional y complejo que comprende la interacción de factores internos, propiamente cognitivos, y externos, del contexto.

2. En el análisis de la definición de metacognición, las dos grandes dimensiones en el concepto son el autoconocimiento de los procesos cognitivos, propios y de los demás; y la autorregulación, tal como sintetizan Pérez y Gonzáles (2020).

3. A manera de síntesis, se propone precisar los procesos cognitivos que el estudiantado debe comprender y ser capaz de gestionar, los cuales se integran en una dinámica holística: motivación, voluntad, inteligencia, memoria, imaginación, modelación, desempeño, socialización y autovaloración cuando se resuelven problemas del contexto.

4. La autorregulación se basa en el autoconocimiento para definir la planificación, autodirección y valoración del proceso de desarrollo de competencias.

5. No se han identificado metodologías específicamente estructuradas para el desarrollo de la metacognición por parte del estudiantado.

6. Por la naturaleza de los procesos internos y del contexto, los siguientes ejes resultan básicos para diseñar una metodología práctica: a. reflexión inicial, sobre los factores cognitivos y externos del proceso de desarrollo de competencias; b. planificación, c. autodirección, d. socialización; e. autoevaluación y f. reflexión final.

7. La integración de los procesos que configuran la metacognición es, a su vez, esencial en todo proceso de aprendizaje, por lo que, lógicamente, la metacognición constituye un factor clave del desarrollo de competencias en cualquier contexto.

8. Cómo promover el conocimiento y regulación de los factores internos para mejorar la calidad del aprendizaje constituye un reto que plantea la necesidad de nuevos modelos metodológicos.

Se recomienda realizar estudios orientados a la elaboración de metodologías específicas, que permitan al estudiantado aplicar la metacognición como factor clave para el desarrollo de competencias. 


\section{REFERENCIAS BIBLIOGRÁFICAS}

Botero, A., Alarcón, I., Palomino, M. y Jiménez, M. (2017). Pensamiento crítico, metacognición y aspectos motivacionales: una educación de calidad. Poiésis, (33), 85-103. https://doi.org/10.21501/16920945.2499

Caeiro-Rodríguez, M. (2018). Aprendizaje Basado en la Creación y Educación Artística: proyectos de aula entre la metacognición y la metaemoción. Arte, Individuo y Sociedad 30(1), 159-177. http://dx.doi.org/10.5209/ARIS .57043

Delors, J. (1999). La Educación encierra un tesoro. Informe a la UNESCO de la Comisión Especial de la Educación para el Siglo XXI. Ediciones UNESCO.

De Zubiría, J. (2013). ¿Cómo diseñar un currículo por competencias?: Fundamentos, lineamientos y estrategias. Cooperativa Editorial Magisterio.

Díaz-Barriga, A. (2006), El enfoque de competencias en a educación. ¿Una alternativa o un disfraz de cambio? Perfiles educativos, 28(111), 7-36. http://www.scielo.org.mx/pdf/peredu/v28n111/n111a2.pdf

Gaeta, M. L., Teruel, M. P. y Orejudo, S. (2012). Aspectos motivacionales, volitivos y metacognitivos del aprendizaje autorregulado. Revista Electrónica de Investigación en Psicología de la Educación, 10(1), 73-94. https://www.red alyc.org/pdf/2931/293123551005.pdf

Gómez, D., Galeano, M. y Jaramillo, C. (2015). El estado del arte: una metodología de investigación. Revista Colombiana de Ciencias Sociales 6(2), 423-442. https://doi.org/10.21501/22161201.1469

Guirao, J., Olmedo, A. y Ferrer, E. (2008). El artículo de revisión. Revista Iberoamericana de Enfermería Comunitaria, 1(1), 1-25.https://www.uv.es/joguigo/castellano/castellano/Investigacion_files/el_articulo_de_revision.pdf

Gonzáles, F. E. (2009). Metacognición y aprendizaje estratégico. Revista Integra Educativa, 2(2), 127-136. http://ww w.scielo.org.bo/scielo.php?pid=S1997-40432009000200005\&script=sci_arttext

Klimenko, O. y Alvares, J. L. (2009). Aprender como aprendo: La enseñanza de estrategias metacognitivas. Educación y Educadores, 12(2). https://biblat.unam.mx/hevila/Educacionyeducadores/2009/vol12/no2/1.pdf

López, F. (1997). Diccionario español-griego-latín. www.culturaclasica.com

Mato, D., Espiñeira, E. y López, V. A. (2017). Impacto del uso de estrategias metacognitivas en la enseñanza de las matemáticas. Perfiles educativos, 39(158), 91-111. http://www.scielo.org.mx/pdf/peredu/v39n158/0185-2698 -peredu-39-158-00091.pdf

Monereo, C., Badia, A., Baixeras, M. V., Boadas, E., Castell'o, M. y Guevara, I. (2001). Ser estratégico y autónomo aprendiendo. Graó.

Morin, E. (1999). Los siete saberes necesarios para la educación del futuro. UNESCO.

Motta, R. (2002), Complejidad, educación y transdisciplinariedad. Polis [En linea],3|2002. http://journals.openedi tion.org/polis/7701

Palacios, P., Álvarez, A., Moreira, M. y Morán, M. (2017). Una mirada al pensamiento crítico en la Educación Superior. EDUMECENTRO, 9(4), 194-206. https://www.medigraphic.com/pdfs/edumecentro/ed-2017/ed174n.pdf

Pérez, G. y Gonzáles, L. M. (2020). Una posible definición de metacognición para la enseñanza de las ciencias. Investigações em Ensino de Ciências, 25(1), 385-404. https://doi.org/10.22600/1518-8795.ienci2020v25n1p3 84

Reyes, H. (2020). Artículos de Revisión. Revista médica de Chile, 148(1), 103-108. https://doi.org/10.4067/S003498872020000100103

Rossi, T., Trevisol, A., dos Santos-Nunes, D., Dapieve-Patias, N. y Von Hohendorff, J. (2020). Autoeficácia geral percebida e motivação para aprender em adolescentes do Ensino Médio. Acta Colombiana de Psicología, 23(1), 254-263. http://doi.org/10.14718/ACP.2020.23.1.12

Rovers, S. F. E., Stalmeijer, R. E., van Merriënboer, J. J. G., Savelberg, H. H. C. M. y de Bruin, A. B. H. (2018). How and Why Do Students Use Learning Strategies? A Mixed Methods Study on Learning Strategies and Desirable Difficulties with Effective Strategy Users. Frontiers in Psychology, 9, 1-12. https://doi.org/10.3389/fpsyg.2018 .02501 
Schleicher, A. (2019). PISA 2018: Insights and Interpretations. Organization for Economic and Cooperation Development.

Sepúlveda, M. S. (2017). Las Competencias Transversales, base del Aprendizaje para Toda la Vida. Universidad $E A N$. https://reposital.cuaieed.unam.mx:8443/xmlui/bitstream/handle/20.500.12579/4905/VE17.0970_3 302-1681.pdf?sequence $=1$

Shannon, S. V. (2008). Using Metacognitive Strategies and Learning Styles to Create Self-Directed Learners. Institute for Learning Styles Journal, 1(1), 14-28. http://www.auburn.edu/academic/cla/ilsrj//Journal\%20Volumes/Fal 1\%202008\%20Volume\%201\%20PDFs/Metacognitive\%20Strategies\%20and\%20Learning\%20Styles.pdf

Sierra, I. (2010). Estrategias de mediación metacognitiva en ambientes convencionales y virtuales: Influencia en los procesos de autorregulación y aprendizaje autónomo en estudiantes universitarios. Editorial de la Universidad de Granada.

Sierra, I. y Carrascal, N. (2005). La gestión del currículo y de los ambientes de aprendizaje para el desarrollo de competencias cognitivas en la formación inicial de educadores en el departamento de Córdoba. Enunciación, 10(1), 31-39. https://revistas.udistrital.edu.co/index.php/enunc/article/view/450/684

Thornberry, G. (2008). Estrategias metacognitivas, motivación académica y rendimiento académico en alumnos ingresantes a una universidad de Lima metropolitana. Persona, (011), 177-193. https://doi.org/10.26439/pers ona2008.n011.935

Tobón, S. (2008). La formación basada en competencias en la educación\# superior: El enfoque complejo. Universidad Autón\#oma de Guadalajara.

Tobón, S. (2013). Metacognicion madfa. (E-book). Plataforma Digital de Publicaciones Issuu. https://issuu.com/cife /docs/e_book_metacognicion_madfa

Tobón, S. (2015a). Formación integral y competencias. Editorial Macro.

Tobón, S. (2015b). Cartografía conceptual. (E-book). Plataforma Digital de Publicaciones Issuu. https://issuu.com/c ife/docs/e-book_cartograf_a_conceptual

Tobón, S., Gonzalez, L., Salvador Nambo, J. y Vazquez Antonio, J. M. (2015). La socioformación: un estudio conceptual. Paradigma, 36(1), 7-29. http://ve.scielo.org/pdf/pdg/v36n1/art02.pdf

Tobón, S., Prieto, J. y Fraile, J. (2010). Secuencias didácticas: Aprendizaje y evaluación de competencias. Pearson educación.

Torrano, F., Fuentes, J., Soria, M. (2017). Aprendizaje autorregulado: Estado de la cuestión y retos psicopedagógicos. Perfiles educativos, 39(156), 160-173. http://www.scielo.org.mx/scielo.php?script=sci_arttext\&pid=S0185-26 982017000200160\&lng=es\&tlng=es.

Valle, A., Regueiro, B., Estévez, I., Piñeiro, I., Rodríguez, S. y Freire, C. (2015). Implicación y motivación hacia los deberes escolares en los estudiantes de primaria según el rendimiento académico y el curso. European Journal of Investigation in Health, Psychology and Education, 5(3), 342-355. https://doi.org/10.1989/ejihpe.v5i3.137

Wisdom, S. y Leavitt, L. (Eds.) (2015). Handbook of Research on Advancing Critical Thinking in Higher Education. IGI Global. https://doi.org/10.4018/978-1-4666-8411-9

\section{INFORMACIÓN ADICIONAL}

Cómo citar: Moreno Muro, J. P., Arbulú Pérez Vargas, C. G. y Montenegro Camacho, L. (2022). La metacognición como factor de desarrollo de competencias en la educación peruana. Revista Educación, 46(1). http://doi.org/10.15517/revedu.v46i1.43724 